\title{
Evaluation of the need for routine feeding jejunostomy for enteral nutrition after esophagectomy
}

\author{
Yuji Akiyama $^{1}$, Takeshi Iwaya ${ }^{1}$, Fumitaka Endo ${ }^{1}$, Haruka Nikai $^{1}$, Kei Sato ${ }^{1}$, Shigeaki Baba ${ }^{1}$, Takehiro Chiba ${ }^{1}$, \\ Toshimoto Kimura ${ }^{1}$, Takeshi Takahara ${ }^{1}$, Hiroyuki Nitta ${ }^{1}$, Koki Otsuka ${ }^{1}$, Masaru Mizuno ${ }^{1}$, Yusuke Kimura ${ }^{2}$, \\ Keisuke Koeda ${ }^{3}$, Akira Sasaki ${ }^{1}$
}

${ }^{1}$ Department of Surgery, Iwate Medical University School of Medicine, Iwate, Japan; ${ }^{2}$ Department of Palliative Medicine, Iwate Medical University School of Medicine, Iwate, Japan; ${ }^{3}$ Department of Medical Safety Science, Iwate Medical University School of Medicine, Iwate, Japan

Contributions: (I) Conception and design: Y Akiyama, T Iwaya, T Kimura, T Takahara, M Mizuno, Y Kimura, K Koeda; (II) Administrative support: K Otsuka, H Nitta, A Sasaki; (III) Provision of study materials or patients: S Baba, T Chiba; (IV) Collection and assembly of data: F Endo, H Nikai, K Sato; (V) Data analysis and interpretation: Y Akiyama, T Iwaya; (VI) Manuscript writing: All authors; (VII) Final approval of manuscript: All authors.

Correspondence to: Yuji Akiyama. Department of Surgery, Iwate Medical University School of Medicine, 19-1 Uchimaru Morioka, Iwate 020-8505, Japan. Email: yakiyama@iwate-med.ac.jp.

Background: Previous studies have shown that enteral nutrition (EN) helps reduce severe postoperative complications after esophagectomy. However, the incidence of jejunostomy-related complications is approximately $30 \%$. We evaluated the operative outcomes in patients who did not receive EN via feeding jejunostomy after esophagectomy.

Methods: We retrospectively reviewed 76 consecutive patients with esophageal cancer who received radical esophagectomy. Operative outcomes were compared between 33 patients who received postoperative EN via feeding jejunostomy (group A; from May 2014 to September 2015) and 43 patients who did not receive EN via feeding jejunostomy (group B; from September 2015 to December 2017).

Results: The American Society of Anesthesiologists performance status score of the patients in group B was significantly higher than that of patients in group $\mathrm{A}(\mathrm{P}=0.002)$. The postoperative morbidity rate was comparable between the two groups (group A, $30.3 \%$ vs. group B, 44.2\%, $\mathrm{P}=0.217$ ). No significant betweengroup differences were observed in the incidence of infectious complications, postoperative hospital stay, readmission within 30 days after discharge, or pneumonia after discharge within 6 months. The incidence of bowel obstruction was significantly higher in group A than in group B (group A, 9.1\% vs. group B, 0\%, $\mathrm{P}=0.044$ ). Two patients in group B required nutritional support via total parenteral nutrition due to bilateral vocal cord palsy or pneumonia.

Conclusions: Jejunostomy-related bowel obstruction in the patients with feeding jejunostomy was significantly higher than that in the patients without jejunostomy. There was no increase in postoperative complications (including pneumonia) in the patients who did not receive EN via feeding jejunostomy. Our results suggest that routine feeding jejunostomy may not be necessary for all patients undergoing esophagectomy.

Keywords: Enteral nutrition (EN); jejunostomy; esophageal cancer; esophagectomy

Submitted Aug 09, 2018. Accepted for publication Nov 15, 2018.

doi: $10.21037 /$ jtd.2018.11.97

View this article at: http://dx.doi.org/10.21037/jtd.2018.11.97 


\section{Introduction}

Radical esophagectomy for esophageal cancer is one of the most invasive surgical procedures of the gastrointestinal tract and is associated with high mortality and morbidity rates $(1,2)$. Postoperative complications, including anastomotic leak, pneumonia, and sepsis, result in prolonged hospital stay and deterioration of physical strength and quality of life (2). Moreover, postoperative complications have been shown to adversely affect overall survival after esophagectomy $(3,4)$. Several studies have shown that enteral nutrition (EN) helps reduce the incidence of severe postoperative complications after esophagectomy $(5,6)$. Furthermore, postoperative early EN has been shown to prevent postoperative weight loss, facilitate early recovery of bowel movements, and reduce length of hospital stay (7-9). In a recent study, EN was shown to ameliorate weight loss and reduce the incidence of pneumonia after thoracoscopic esophagectomy, a minimally invasive surgery (10). On the other hand, jejunostomy-related complications have a relatively high incidence (28.5-38\%) (11-13). In a study by Han-Geurts et al., infection at the insertion site was the most common complication (16\%), followed by catheter dislocation $(6 \%)$, obstruction $(6 \%)$, removal by patients (5\%), and leakage (4\%) (11). Leakage or intestinal obstruction often necessitates relaparotomy $(11,13)$. Bowel obstruction related to jejunostomy occurred even after jejunal tube removal and often required operation. The jejunal tube is typically not removed at discharge in patients who do not develop complications; therefore, self-care of the tube at home by these patients is required. In-dwelling tubes or catheters tend to hinder physical activities after surgery. Therefore, we discontinued the practice of routine EN via feeding jejunostomy after esophagectomy. In this study, we investigated the operative outcomes of patients with esophageal cancer who did not receive EN via feeding jejunostomy after esophagectomy.

\section{Methods}

\section{Patients}

We retrospectively reviewed 76 consecutive patients with esophageal squamous cell carcinoma who underwent radical esophagectomy at the Department of Surgery, Iwate Medical University Hospital, between May 2014 and December 2017. The practice of postoperative EN via feeding jejunostomy was discontinued in September 2015. Operative outcomes were compared between patients who received postoperative EN via feeding jejunostomy (group A; from May 2014 to September 2015) and patients who did not receiving postoperative $\mathrm{EN}$ via feeding jejunostomy (group B; from September 2015 to December 2017). The clinical characteristics of the patients are presented in Table 1. The tumor location was classified according to the Japanese Classification of Esophageal Cancer (JCEC), 11th edition (14). Clinical stage was determined according to the Union for International Cancer Control classification, 7th edition. Neoadjuvant chemotherapy with cisplatin and fluorouracil was offered to patients with stage II/III squamous cell carcinoma, based on the standard preoperative treatment in Japan (15). Patients with more advanced cancer, including those with severe lymph node metastasis (mainly exceeding stage III) received first-line chemotherapy with docetaxel, cisplatin, and fluorouracil, as previously reported (16).

Radical esophagectomy via right thoracotomy or thoracoscopy was performed according to the guidelines for the diagnosis and treatment of carcinoma of the esophagus in Japan (17). Thoracoscopic esophagectomy was performed in the prone position as previously reported $(18,19)$. In most cases, the reconstruction conduit was a gastric tube pulled through the posterior mediastinum or via the retrosternal route with cervical esophagogastrostomy (20). In group A, a jejunostomy was placed $20 \mathrm{~cm}$ distal from the ligament of Treitz using the 9 Fr Kangaroo jejunostomy catheter kit ${ }^{\circledR}$ (Medtronic, Minneapolis, MN, USA) under laparotomy. A jejunal tube was inserted $4 \mathrm{~cm}$ along the subserosa and then into the intestinal lumen for a total of $40 \mathrm{~cm}$. The tube was attached to the jejunum using a purse string suture and was fixed to the anterior abdominal wall in a horizontal orientation for $5 \mathrm{~cm}$ with additional six sutures.

The perioperative management of radical esophagectomy has been described previously (21). In brief, patients received rehabilitation before surgery and started an early mobilization with ambulation, respiratory training, and training for chewing and swallowing on postoperative day (POD) 1. All patients stayed in the intensive care unit until POD 4 . When the patients were moved to a surgical ward on POD 4, they were able to walk freely within the hospital. After POD 7, patients were trained in the rehabilitation room to recover muscle strength and used an ergometer. The nasogastric tube was removed on the morning of POD 1. The chest drain was routinely removed on POD 4 in the absence of any chyle leak. The urinary catheter was also routinely removed on POD 4. Patients were discharged when they were able to achieve stable oral intake. 
Table 1 Clinical characteristics of the study population

\begin{tabular}{|c|c|c|c|}
\hline Characteristics & $\begin{array}{c}\text { Group A } \\
(\mathrm{N}=33)\end{array}$ & $\begin{array}{c}\text { Group B } \\
(\mathrm{N}=43)\end{array}$ & $P$ value \\
\hline $\mathrm{Age}^{\mathrm{a}}$ (years) & $66.2 \pm 10.4$ & $66.7 \pm 8.6$ & 0.812 \\
\hline Sex & & & 0.894 \\
\hline Male & 28 & 36 & \\
\hline Female & 5 & 7 & \\
\hline Histologic type ${ }^{b}$ & & & 0.668 \\
\hline Squamous cell carcinoma & 32 & 41 & \\
\hline Adenocarcinoma & 1 & 1 & \\
\hline Malignant melanoma & 0 & 1 & \\
\hline Tumor location ${ }^{\mathrm{b}}$ & & & 0.787 \\
\hline Ut & 3 & 7 & \\
\hline Mt & 21 & 24 & \\
\hline $\mathrm{Lt}$ & 8 & 10 & \\
\hline $\mathrm{Ae}$ & 1 & 2 & \\
\hline Clinical stage ${ }^{c}$ & & & 0.652 \\
\hline IA & 9 & 16 & \\
\hline IB & 3 & 2 & \\
\hline IIA & 2 & 2 & \\
\hline IIB & 4 & 1 & \\
\hline IIIA & 6 & 9 & \\
\hline IIIB & 2 & 4 & \\
\hline IIIC & 7 & 8 & \\
\hline IV & 0 & 1 & \\
\hline Pathological stage $^{c}$ & & & 0.194 \\
\hline 0 & 1 & 10 & \\
\hline IA & 12 & 12 & \\
\hline IB & 3 & 3 & \\
\hline IIA & 2 & 3 & \\
\hline IIB & 6 & 5 & \\
\hline IIIA & 6 & 4 & \\
\hline IIIC & 3 & 3 & \\
\hline IV & 0 & 3 & \\
\hline Preoperative treatment & & & 0.262 \\
\hline No & 13 & 18 & \\
\hline Yes & 20 & 25 & \\
\hline CF & 0 & 4 & \\
\hline DCF & 18 & 20 & \\
\hline CRT & 2 & 1 & \\
\hline
\end{tabular}

Table 1 (continued)

\begin{tabular}{lccc}
\hline Characteristics & $\begin{array}{c}\text { Group A } \\
(\mathrm{N}=33)\end{array}$ & $\begin{array}{c}\text { Group B } \\
(\mathrm{N}=43)\end{array}$ & P value \\
\hline ASAPS & 9 & 2 & 0.002 \\
1 & 24 & 33 & \\
2 & 0 & 8 & \\
3 & & & \\
Comorbidities & 18 & 19 & 0.371 \\
Heart disease & 2 & 3 & 0.873 \\
Pulmonary disease & 7 & 13 & 0.376 \\
$\quad$ Diabetes mellitus & $22.3 \pm 3.3$ & $22.2 \pm 2.9$ & 0.906 \\
Body mass index ${ }^{\mathrm{a}}\left(\mathrm{kg} / \mathrm{m}^{2}\right)$ & $6.5 \pm 0.5$ & $6.7 \pm 0.7$ & 0.218 \\
Total protein $^{\mathrm{a}}(\mathrm{g} / \mathrm{dL})$ & $3.9 \pm 0.5$ & $4.1 \pm 0.4$ & 0.204 \\
Albumin $^{\mathrm{a}}$ (g/dL) & & & 0.088 \\
Surgical procedure $^{2}$ & & &
\end{tabular}

Surgical procedure

$\begin{array}{lr}4 & 1 \\ 29 & 42\end{array}$

Thoracoscopy
Reconstruction conduit

0.251

Gastric tube $\quad 32 \quad 43$

Colon

Route of reconstruction

0.505

Posterior mediastinal

Retrosternal

Anterior sternal

Lymph node dissection

0.092

Two-field

Three-field

$$
21
$$

24

19

Abdominal approach

0.043

\begin{tabular}{lcc} 
Laparotomy & 14 & 9 \\
HALS & 19 & 34 \\
\hline
\end{tabular}

${ }^{a}$, mean \pm standard deviation; ${ }^{b}$, according to the Japanese Classification of Esophageal Cancer, 11th edition (Ut: upper thoracic esophagus, Mt: middle thoracic esophagus, Lt: lower thoracic esophagus, and Ae: abdominal esophagus); ${ }^{\text {c }}$, according to the Union for International Cancer Control classification, 7th edition. ASAPS, American Society of Anesthesiologists performance states; CF, cisplatin and 5-fluorouracil; DCF, docetaxel, cisplatin, and 5-fluorouracil; CRT, chemoradiotherapy; HALS, hand-assisted laparoscopic surgery.

Table 1 (continued) 


\section{Nutritional treatment}

Postoperatively, all patients received continuous infusion of Ringer's acetate solution on the operative day $(5 \mathrm{~mL} / \mathrm{kg} / \mathrm{h})$ and amino acid solution with $7.5 \%$ glucose (BFLUID ${ }^{\circledR}$; Otsuka Pharmaceutical Factory, Inc., Tokushima, Japan) on PODs 1-7 (approximately $2 \mathrm{~mL} / \mathrm{kg} / \mathrm{h} ; 15 \mathrm{kcal} / \mathrm{kg} /$ day) through peripheral intravenous catheters. Oral intake was started on POD 5 or 6 after evaluation of recurrent laryngeal nerve paralysis by video endoscopic examination in each group.

In group A, continuous enteral feeding via jejunostomy was started on POD 1 at the rate of $5 \mathrm{kcal} / \mathrm{kg} /$ day; the quantity of nutrition was progressively increased by $5 \mathrm{kcal} / \mathrm{kg} /$ day up to $30 \mathrm{kcal} / \mathrm{kg} /$ day on POD 6 . EN feeding was gradually decreased based on the status of oral ingestion to achieve a total energy intake of $30 \mathrm{kcal} / \mathrm{kg} / \mathrm{day}$. The jejunal tube was removed in the outpatient department approximately 1-2 months after surgery without usage at home.

\section{Outcome measures}

Postoperative morbidity and mortality rates, postoperative laboratory data, loss of body weight, hospitalization, and readmission within 30 days after surgery, readmission due to malnutrition, and pneumonia after discharge within 6 months were compared between the two groups. Postoperative laboratory investigations included white blood cell counts, C-reactive protein, total bilirubin, aspartate transaminase, alanine aminotransferase, blood urea nitrogen, and creatinine at maximal values within POD 7 , and total protein and albumin at POD 14. Data pertaining to body weight loss were collected at POD 14 and at 3 and 6 months after surgery. Postoperative complications were defined based on the classification of the Esophagectomy Complications Consensus Group (2). Clostridium difficile (C. difficile) enteritis was defined as the presence of enteritis by laboratory detection of $C$. difficile-positive toxin in the stool or a $C$. difficile-positive stool culture. Complication grades were defined according to the Clavien-Dindo classification (22).

\section{Statistical analysis}

Statistical analyses were performed using the SAS statistical analysis software, JMP 10 (SAS, Cary, NC, USA). Betweengroup differences in patient characteristics and outcomes were assessed using the chi-squared test, Student's $t$ test, or Wilcoxon's rank test. $\mathrm{P}<0.05$ was considered statistically significant.

\section{Results}

The clinical characteristics of the patients are summarized in Table 1. Of the 76 reviewed patients with esophageal cancer, 64 were men $(84.2 \%)$ and 12 women $(15.8 \%)$ with a mean age of 66.4 (age range: $33-84$ ) years. The major histological type was squamous cell carcinoma (96\%). In total, 45 patients $(59.2 \%)$ were treated with preoperative chemotherapy or chemoradiotherapy. In most cases, thoracoscopic esophagectomy was performed (group A, $87.9 \%$ vs. group B, $97.7 \%$ ) via gastric tube reconstruction through the posterior mediastinum. No significant betweengroup differences were observed with respect to age, sex ratio, body mass index, histologic types, tumor location, clinical and pathological stage, preoperative treatment, comorbidities, or surgical procedures. The American Society of Anesthesiologists performance status (ASAPS) of patients in group $B$ was significantly higher than that of patients in group $\mathrm{A}(\mathrm{P}=0.002$, Table 1).

Postoperative morbidity is presented in Table 2. Morbidity rate in groups A and B was $30.3 \%$ and $44.2 \%$, respectively; the between-group difference was not statistically significant (Table 2). There was no significant between-group difference in the overall rate of infectious complications (including pneumonia, wound infection, bacteremia, or $C$. difficile enteritis) (group A, $21.2 \%$ vs. group B, 18.6\%, Table 2). EN administration was temporarily stopped in two patients in group A due to diarrhea caused by $C$. difficile enteritis; EN was restarted after improvement in its symptoms. Other patients in group A were administered $\mathrm{EN}$ as per schedule. Operative mortality was zero in both groups. The incidence of bowel obstruction was significantly higher in group A (group A, 9.1\% vs. group B, 0\%, $\mathrm{P}=0.044$, Table 2). All cases of bowel obstruction occurring after discharge were associated with jejunostomy and necessitated reoperation.

The postoperative outcomes in groups A and B are shown in Table 3. No significant between-group differences were observed with respect to postoperative blood investigations, start of oral intake after surgery, length of postoperative hospital stay, readmission within 30 days after discharge, readmission due to malnutrition, or pneumonia after discharge within 6 months (Table 3). The rate of weight loss on POD 14 in group B was significantly higher than that in group A (group A, $1.4 \%$ vs. group B, $5.1 \%, \mathrm{P}<0.001$, Table 3). However, there were no significant betweengroup differences in the rate of weight loss at 3 months and 6 months after surgery. Two patients in group B required nutritional support via total parenteral nutrition (TPN) due 
Table 2 Postoperative morbidity

\begin{tabular}{|c|c|c|c|}
\hline Complications & $\begin{array}{c}\text { Group A } \\
(\mathrm{N}=33)\end{array}$ & $\begin{array}{l}\text { Group B } \\
(\mathrm{N}=43)\end{array}$ & $P$ value \\
\hline Morbidity & $10(30.3)$ & $19(44.2)$ & 0.217 \\
\hline Pneumonia & $4(12.1)$ & 5 (11.6) & 0.947 \\
\hline Dysrhythmia atrial & 0 & $3(7.0)$ & 0.122 \\
\hline Anastomotic leak & 0 & 0 & - \\
\hline Conduit necrosis & 0 & $1(2.3)$ & 0.378 \\
\hline Chyle leak & 0 & 0 & - \\
\hline Vocal cord palsy & $3(9.1)$ & $8(18.6)$ & 0.243 \\
\hline $\begin{array}{l}\text { Bleeding requiring } \\
\text { reoperation }\end{array}$ & 0 & $1(2.3)$ & 0.378 \\
\hline Wound infection & $1(3.0)$ & 0 & 0.251 \\
\hline $\begin{array}{l}\text { Clostridium difficile } \\
\text { enteritis }\end{array}$ & $2(6.1)$ & $2(4.7)$ & 0.785 \\
\hline Bacteremia & 0 & $1(2.3)$ & 0.378 \\
\hline Bowel obstruction & $3(9.1)$ & 0 & 0.044 \\
\hline Overall infectious disease & 7 (21.2) & $8(18.6)$ & 0.777 \\
\hline $\begin{array}{l}\text { Clavien-Dindo } \\
\text { classification }\end{array}$ & & & 0.755 \\
\hline I & 4 & 6 & \\
\hline II & 6 & 11 & \\
\hline IIla & 0 & 1 & \\
\hline IIIb & 0 & 1 & \\
\hline Mortality & 0 & 0 & - \\
\hline
\end{tabular}

to bilateral vocal cord palsy or pneumonia (Table 3).

\section{Discussion}

Several studies have shown that the use of EN in the early postoperative period after esophagectomy more effectively reduced postoperative complications than parenteral nutrition (5-10). Moreover, EN was better than parenteral nutrition support for maintaining nutritional status and prevention of postoperative weight loss $(5,7,10)$. EN also reduced the duration of intensive care unit treatment and the total hospital stay $(8,9)$. Reportedly, early EN is one of the factors that improves immune function and attenuates changes in cytokine levels after esophagectomy $(23,24)$. Meta-analyses revealed that postoperative EN was associated with decreased incidence of severe infectious complications (such as anastomotic leak and pulmonary complications) and shorter length of hospital stay as compared with that with the use of parenteral nutrition $(5,6)$. Feeding jejunostomy was most commonly used for postoperative early EN $(11,13,25)$. In agreeable situations, we also performed early EN after esophagectomy and achieved stable postoperative outcomes $(26,27)$. However, feeding jejunostomy itself is vulnerable to complications such as obstruction, leakage, and dislocation (11-13). These complications sometimes necessitate reoperation. In this study, bowel obstruction related to jejunostomy occurred in three patients after discharge (Table 2). Table 4 depicts characteristics of patients who developed bowel obstruction related to jejunostomy in this series; reoperation was required in all instances. The median duration between esophagectomy and the onset of bowel obstruction was 238 (range: 191-281) days; patients required long-term care after esophagectomy. Causes of jejunostomy-related bowel obstruction included occlusion or torsion of the jejunum at the site of insertion of the jejunostomy tube, which was caused by separation of the fixation from the jejunum and abdominal wall. This problem occurred despite the use of a non-absorbable thread for fixation in this series. Therefore, it may be necessary to additionally secure the fixation between the jejunum and abdominal wall. Moreover, some cases developed occlusion of the feeding tube (13). It is difficult to regulate the speed of administration of EN via jejunostomy, and this may cause abdominal discomfort or pain. An infusion pump is required for continuous administration of EN, which hinders the physical activity of patients. Since the jejunal tube is not removed at discharge, the patients are required to take care of tube at home. Owing to these demerits of feeding jejunostomy, we discontinued the practice of EN via feeding jejunostomy after esophagectomy.

The recent introduction of perioperative enhanced recovery protocol implemented by a multidisciplinary support team has helped improve the operative outcomes of esophagectomy at our hospital (21). In this study, the performance status (ASAPS) of patients in group B (after discontinuation of EN via jejunostomy) was significantly higher than that in group A (before discontinuation of EN) (Table 1). This reflects the change in institutional protocol over the course of the study reference period by which the indications for esophagectomy were extended to include high-risk patients after stabilization of outcomes. However, there was no increase in the incidence of postoperative complications or prolongation of length of hospital stay after 
Table 3 Postoperative outcomes

\begin{tabular}{|c|c|c|c|}
\hline Outcomes & Group A (N=33) & Group B (N=43) & $\mathrm{P}$ value \\
\hline $\mathrm{WBC}^{\mathrm{b}}(/ \mu \mathrm{L})$ & $13,744.6 \pm 7,531.7$ & $12,698.6 \pm 3,003.4$ & 0.456 \\
\hline $\mathrm{CRP}^{\mathrm{b}}(\mathrm{mg} / \mathrm{dL})$ & $9.1 \pm 4.5$ & $10.1 \pm 7.1$ & 0.470 \\
\hline Total protein ${ }^{c}(\mathrm{~g} / \mathrm{dL})$ & $6 \pm 0.6$ & $6.1 \pm 0.4$ & 0.174 \\
\hline Total bilirubin ${ }^{\mathrm{b}}(\mathrm{mg} / \mathrm{dL})$ & $0.8 \pm 0.5$ & $1 \pm 0.6$ & 0.168 \\
\hline $\mathrm{AST}^{\mathrm{b}}(\mathrm{U} / \mathrm{L})$ & $62.7 \pm 33.9$ & $53.3 \pm 22.7$ & 0.175 \\
\hline $\mathrm{ALT}^{\mathrm{b}}(\mathrm{U} / \mathrm{L})$ & $64.4 \pm 54$ & $45.9 \pm 27$ & 0.079 \\
\hline $\mathrm{BUN}^{\mathrm{b}}(\mathrm{mg} / \mathrm{dL})$ & $22.5 \pm 7.7$ & $22.2 \pm 4$ & 0.835 \\
\hline \multicolumn{4}{|l|}{ Rate of weight loss ${ }^{\mathrm{a}}(\%)$} \\
\hline POD 14 & $1.4 \pm 4.4$ & $5.1 \pm 3.4$ & $<0.001$ \\
\hline POM 3 & $7.4 \pm 5.5$ & $7.5 \pm 4.6$ & 0.951 \\
\hline POM 6 & $9.1 \pm 6.4$ & $8.6 \pm 5.9$ & 0.788 \\
\hline Postoperative TPN use & 0 & 2 & 0.209 \\
\hline Postoperative hospital stay ${ }^{a}$ (days) & $20.2 \pm 4.6$ & $17.8 \pm 5.7$ & 0.055 \\
\hline Readmission within 30 days after discharge & 0 & 0 & - \\
\hline Readmission due to malnutrition & 0 & 0 & - \\
\hline
\end{tabular}

${ }^{a}$, mean \pm standard deviation; ${ }^{b}$, maximal values within POD $7 ;{ }^{c}$, values at POD $14 ;{ }^{d}$, pneumonia in the first 6 months after discharge. AST, aspartate transaminase; ALT, alanine aminotransferase; BUN, blood urea nitrogen; CRP, C-reactive protein; POD, postoperative day; POM, postoperative month; TPN, total parenteral nutrition; WBC, white blood cell.

Table 4 Characteristics of patients who developed feeding jejunostomy-related bowel obstruction and their treatment details

\begin{tabular}{lllllllll}
\hline Case & Gender & Age & $\begin{array}{l}\text { Operative method in } \\
\text { esophagectomy }\end{array}$ & $\begin{array}{l}\text { Abdominal } \\
\text { approach }\end{array}$ & $\begin{array}{l}\text { Complication after } \\
\text { esophagectomy }\end{array}$ & $\begin{array}{c}\text { Duration } \\
\text { (days) }\end{array}$ & $\begin{array}{l}\text { Operative }_{\text {findings }} \\
\text { Operative procedure }\end{array}$ \\
\hline 1 & Male & 68 & Thoracoscopy & HALS & Vocal code palsy & 243 & Occlusion & Release of occlusion \\
2 & Male & 74 & Thoracoscopy & HALS & lleus with enteritis & 191 & Torsion & Release of torsion \\
3 & Male & 70 & Thoracoscopy & HALS & None & 281 & Occlusion & Release of occlusion \\
\hline
\end{tabular}

${ }^{\mathrm{a}}$, duration between esophagectomy and onset of bowel obstruction. HALS, Hand-assisted laparoscopic surgery.

discontinuation of the practice of EN. In this study, body weight loss at POD 14 in patients who did not receive EN via jejunostomy was significantly higher than that in patients who received EN. However, this did not lead to prolongation of hospital stay, and there was no significant between- group difference in body weight loss at 3 months after esophagectomy. None of the patients who did not receive EN required readmission due to malnutrition (Table 3).

Postoperative EN is preferred in patients who develop complications (such as bilateral vocal cord palsy, severe 
respiratory complications, or anastomotic leak) that necessitate nil oral intake over a prolonged period. In our study, 2 of 43 patients (4.7\%) were administered TPN due to bilateral vocal cord palsy or pneumonia in group B (Table 3). Although previous studies have investigated the factors associated with postoperative complications (28-30), accurately predicting surgical outcomes before surgery is difficult in clinical practice. Patients with respiratory comorbidities or decreasing preoperative respiratory function may not always develop postoperative pulmonary complications. Practically, jejunostomy should be performed for elderly patients with reduced function of swallowing preoperatively and for patients with intraoperatively confirmed bilateral vocal code palsy or depressed bloodstream of conduit $(31,32)$. If complications arise after esophagectomy, nasojejunal tube can be selected. The nasojejunal tube was shown to be a feasible route for EN (11,33). However, prolonged use of the nasojejunal tube causes discomfort and hinders swallowing. In cases where long-term EN is necessary (e.g., patients with complications that necessitate nil oral intake or malnourished patients), jejunostomy should be performed after surgery. On the other hand, early oral feeding should be considered if the postoperative course is uneventful $(34,35)$.

The present study shows that postoperative treatment without EN via feeding jejunostomy is feasible and does not increase postoperative complications. However, there are limitations to our study. First, this was a retrospective cohort study with a small study population. Second, early oral intake was possible in most patients in this series due to lack of complications. The rate of anastomotic leak was relatively low (20). None of the patients developed anastomotic leak in this study.

\section{Conclusions}

Jejunostomy-related bowel obstruction in patients with feeding jejunostomy was significantly higher than that in patients without jejunostomy. There was no increase in postoperative complications, including pneumonia, in patients who did not receive EN via feeding jejunostomy. Our results suggest that routine feeding jejunostomy may not be necessary for all patients undergoing esophagectomy.

\section{Acknowledgements}

We thank all the participants of this study and the nursing and laboratory staff of Iwate Medical University Hospital.

\section{Footnote}

Conflicts of Interest: The authors have no conflicts of interests to declare.

Ethical Statement: This retrospective study protocol was reviewed and approved by the ethical committee of the Iwate Medical University, School of Medicine (No. MH2018-560).

\section{References}

1. Markar S, Gronnier C, Duhamel A, et al. Pattern of postoperative mortality after esophageal cancer resection according to center volume: Results from a large european multicenter study. Ann Surg Oncol 2015;22:2615-23.

2. Low DE, Alderson D, Cecconello I, et al. International consensus on standardization of data collection for complications associated with esophagectomy: Esophagectomy Complications Consensus Group (ECCG). Ann Surg 2015;262:286-94.

3. Kataoka K, Takeuchi H, Mizusawa J, et al. Prognostic impact of postoperative morbidity after esophagectomy for esophageal cancer: Exploratory analysis of JCOG9907. Ann Surg 2017;265:1152-7.

4. Baba Y, Yoshida N, Shigaki H, et al. Prognostic Impact of Postoperative complications in 502 patients with surgically resected esophageal squamous cell carcinoma: A retrospective single-institution study. Ann Surg 2016;264:305-11.

5. Peng J, Cai J, Niu ZX, et al. Early enteral nutrition compared with parenteral nutrition for esophageal cancer patients after esophagectomy: a meta-analysis. Dis Esophagus 2016;29:333-41.

6. Mazaki T, Ebisawa K. Enteral versus parenteral nutrition after gastrointestinal surgery: a systematic review and meta-analysis of randomized controlled trials in the English literature. J Gastrointest Surg 2008;12:739-55.

7. Ryan AM, Rowley SP, Healy LA, et al. Postoesophagectomy early enteral nutrition via a needle catheter jejunostomy: 8-year experience at a specialist unit. Clin Nutr 2006;25:386-93.

8. Gabor S, Renner H, Matzi V, et al. Early enteral feeding compared with parenteral nutrition after oesophageal or oesophagogastric resection and reconstruction. Br J Nutr 2005;93:509-13.

9. Barlow R, Price P, Reid TD, et al. Prospective multicentre randomised controlled trial of early enteral nutrition for 
patients undergoing major upper gastrointestinal surgical resection. Clin Nutr 2011;30:560-6.

10. Takesue T, Takeuchi H, Ogura M, et al. A prospective randomized trial of enteral nutrition after thoracoscopic esophagectomy for esophageal cancer. Ann Surg Oncol 2015;22:S802-9.

11. Han-Geurts IJ, Hop WC, Verhoef C, et al. Randomized clinical trial comparing feeding jejunostomy with nasoduodenal tube placement in patients undergoing oesophagectomy. Br J Surg 2007;94:31-5.

12. Torres Júnior LG, de Vasconcellos Santos FA, Correia MI. Randomized clinical trial: nasoenteric tube or jejunostomy as a route for nutrition after major upper gastrointestinal operations. World J Surg 2014;38:2241-6.

13. Weijs TJ, van Eden HWJ, Ruurda JP, et al. Routine jejunostomy tube feeding following esophagectomy. J Thorac Dis 2017;9:S851-60.

14. Japan Esophageal Society. Japanese classification of esophageal cancer. 11th ed. Tokyo: Kanehara; 2015.

15. Ando N, Kato H, Igaki H, et al. A randomized trial comparing postoperative adjuvant chemotherapy with cisplatin and 5-fluorouracil versus preoperative chemotherapy for localized advanced squamous cell carcinoma of the thoracic esophagus (JCOG9907). Ann Surg Oncol 2012;19:68-74.

16. Akiyama Y, Iwaya T, Endo F, et al. Investigation of operative outcomes of thoracoscopic esophagectomy after triplet chemotherapy with docetaxel, cisplatin, and 5-fluorouracil for advanced esophageal squamous cell carcinoma. Surg Endosc 2018;32:391-9.

17. Kuwano H, Nishimura Y, Oyama T, et al. Guidelines for diagnosis and treatment of carcinoma of the esophagus April 2012 edited by the Japan Esophageal Society. Esophagus 2015;12:1-30.

18. Noshiro H, Iwasaki H, Kobayashi K, et al.

Lymphadenectomy along the left recurrent laryngeal nerve by a minimally invasive esophagectomy in the prone position for thoracic esophageal cancer. Surg Endosc 2010;24:2965-73.

19. Ozawa S, Ito E, Kazuno A, et al. Thoracoscopic esophagectomy while in a prone position for esophageal cancer: a preceding anterior approach method. Surg Endosc 2013;27:40-7.

20. Akiyama Y, Iwaya T, Endo F, et al. Stability of cervical esophagogastrostomy via hand-sewn anastomosis after esophagectomy for esophageal cancer. Dis Esophagus 2017;30:1-7.

21. Akiyama Y, Iwaya T, Endo F, et al. Effectiveness of intervention with a perioperative multidisciplinary support team for radical esophagectomy. Support Care Cancer 2017;25:3733-9.

22. Dindo D, Demartines N, Clavien PA. Classification of surgical complications: a new proposal with evaluation in a cohort of 6336 patients and results of a survey. Ann Surg 2004;240:205-13.

23. Chen L, Sun L, Lang Y, et al. Fast-track surgery improves postoperative clinical recovery and cellular and humoral immunity after esophagectomy for esophageal cancer. BMC Cancer 2016;16:449.

24. Okamura A, Takeuchi H, Matsuda S, et al. Factors affecting cytokine change after esophagectomy for esophageal cancer. Ann Surg Oncol 2015;22:3130-5.

25. Berkelmans GH, van Workum F, Weijs TJ, et al. The feeding route after esophagectomy: a review of literature. J Thorac Dis 2017;9:S785-S791.

26. Sato N, Koeda K, Ikeda K, et al. Randomized study of the benefits of preoperative corticosteroid administration on the postoperative morbidity and cytokine response in patients undergoing surgery for esophageal cancer. Ann Surg 2002;236:184-90.

27. Ito N, Iwaya T, Ikeda K, et al. Hyperglycemia 3 days after esophageal cancer surgery is associated with an increased risk of postoperative infection. J Gastrointest Surg 2014;18:1547-56.

28. Zingg U, Smithers BM, Gotley DC, et al. Factors associated with postoperative pulmonary morbidity after esophagectomy for cancer. Ann Surg Oncol 2011;18:1460-8.

29. Law S, Wong KH, Kwok KF, et al. Predictive factors for postoperative pulmonary complications and mortality after esophagectomy for cancer. Ann Surg 2004;240:791-800.

30. Ferguson MK, Durkin AE. Preoperative prediction of the risk of pulmonary complications after esophagectomy for cancer. J Thorac Cardiovasc Surg 2002;123:661-9.

31. Hikage M, Kamei T, Nakano T, et al. Impact of routine recurrent laryngeal nerve monitoring in prone esophagectomy with mediastinal lymph node dissection. Surg Endosc 2017;31:2986-96.

32. Koyanagi K, Ozawa S, Oguma J, et al. Blood flow speed of the gastric conduit assessed by indocyanine green fluorescence: New predictive evaluation of anastomotic leakage after esophagectomy. Medicine (Baltimore) 2016;95:e4386.

33. Elshaer M, Gravante G, White J, et al. Routes of early enteral nutrition following oesophagectomy. Ann R Coll Surg Engl 2016;98:461-7. 
34. Sun HB, Li Y, Liu XB, et al. Early oral feeding following McKeown minimally invasive esophagectomy: An openlabel, randomized, controlled, noninferiority trial. Ann Surg 2018;267:435-42.

Cite this article as: Akiyama Y, Iwaya T, Endo F, Nikai H, Sato K, Baba S, Chiba T, Kimura T, Takahara T, Nitta H, Otsuka K, Mizuno M, Kimura Y, Koeda K, Sasaki A. Evaluation of the need for routine feeding jejunostomy for enteral nutrition after esophagectomy. J Thorac Dis 2018;10(12):6854-6862. doi: 10.21037/jtd.2018.11.97
35. Weijs TJ, Berkelmans GH, Nieuwenhuijzen GA, et al. Immediate postoperative oral nutrition following esophagectomy: A multicenter clinical trial. Ann Thorac Surg 2016;102:1141-8. 